\title{
CHARACTERIZATION OF TYPICAL MULTI-PROTOCOL LABEL SWITCHING IN VIRTUAL PRIVATE NETWORK INFRASTRUCTURE IN NIGERIA
}

\author{
Ogbu, Mary N.C., ${ }^{1}$ Onoh, Greg N., ${ }^{1}$ and Abonyi, Dorathy O. ${ }^{1}$ \\ ${ }^{1}$ Department of Electrical and Electronics Engineering, \\ Enugu State of University of Science and Technology Enugu, Nigeria.
}

\begin{abstract}
The performance of the Multi-Protocol Label Switching in Virtual Private Network (MPLSVPN) has been deteriorating because of poor network metrics. This paper illustrated the principal characteristics of the MPLS- enabled systemin four different Virtual Private Networks in Nigeria. Deductions obtained from the measurement shows that Joint Node Location problem and Link Connectivity problem cause the deterioration of the network performance in respect to Quality of Service (QoS).A comparative analysis of the network metrics for Voice over IPwith ITU QoS standard as a benchmark was carried out. The results shows thatMPLS networks in Nigeria has poor performance due decrease in throughput and relatively high delay and packet loss values. Hence there is need for an improvement.
\end{abstract}

Keywords:MPLS, Virtual Private Network, Link Connectivity Problem, Joint Node Link Problem, Internet Service Provider

\section{INTRODUCTION:}

Data communication is one of the most important areas in any developed nation in respect to communication system. Majority of enterprise organizations need a rich IP-based technology that will perform very well. Evolution of the Multi-Protocol Label Switchingby Internet Engineering task Force (IETF) has opened several implementation of this transmission technology to telecommunication system. However, some unavoidable problems affects the connection and optimum performance of MPLS technology. To characterize a cloud-based MPLS-VPN infrastructure for VPN service provisioning, fieldmeasurements were carried out on four different VPN networks in Nigeria. The four typical enterprise Virtual Private Networks were Internet Solution of Nigeria (ISN), Swift Enterprise Limited, University of Nigeria Nsukka (UNN) VPN and Nigeria National Petroleum Corporation VPN. Theinternal structure of their network topologies was studied.

Cole, Thain and Fallon (2015) discussed a small scale laboratory network model used to investigate the performance of MPLS networks while teaching some students in the college. The authors used both OPNET and GNS3 tools to simulate the concept. Later on, the work was conducted in a laboratory experimental setup that involved only physical hardware for the students to have a comprehensive understanding of MPLS concept. Mamum, Sheltami, Ali, et al. (2016) evaluated the performance of video conferencing application using two different routing protocol over an MPLS-VPN network. Open Shortest Path First (OSPF) and Enhanced Interior Gateway Routing Protocol (EIGRP) were the routing protocols. Kale and Waichol (2014) gave a comprehensive performance analysis of MPLS and IP networks in laboratory using Huawei NE40 series as the physical hardware device unlike CISCO and AlCATEL devices other researchers normally used. Wireshark network analyzer and monitoring tools were used to analyze the performance of the MPLS-enabled network in terms of packet loss and throughput. Ahmad et.alpresented a QoS performance analytical 
study of applications like video conferencing, mail, voice and web over DiffServ with MPLS in IPv4 and IPv6 networks using Optimized Network Engineering Tool (OPNET). The performance of the network was analyzed using two virtual core network environment (i.e. MPLS over core IPv4 and IPv6 network). The network topology used to carry out the simulation contains three routers as LER, five routers as LSR, two servers for FTP and database under four different applications (video, voice, data and FTP). (Ahmad, S., Hamdani, W. A., Magray, M. H. 2015).

Almofary, Moustafa and Zaki (2013) explained the concept of Border Gateway protocol in MPLS-VPN in respect to the scalability. The authors used OPNET modeler to study how Route Reflector (RR) can be used to solve scalability problem in a network. Full mesh BGP topology was used between PE routers to enable MPLS and also RR. After simulation, MPLS-VPN backbone including IGP with or without RR were compared. The results showed that the one with Route Reflector improved very well.Abdul-Bary and Omar-Kh (2014); Aggarwal and Dhall (2015) studied the performance measures of an MPLS-TE network and traditional IP network in relation to real time multimedia (video conferencing and voice over IP) and non-real time applications (FTP). Their work were characterized for both MPLS and traditional IP networks in different scenarios which are MPLS network with Traffic Engineering and IP network without Traffic Engineering. Efendi, R. (2012) carried out simulation work to analyze how network parameters like latency and packet loss appear when multi-virtual routing and forwarding were implemented in an MPLS-VPN network. It was observed that the latency of data transmission and the percentage of packet loss were very small especially when IPSec tunneling were enabled in the network. And these metrics could not be increase by encapsulation and encryption process. Kundu, R., Kumar, A. and Wason, A. (2014) proposed and developed an advanced routing algorithm that improved available bandwidth utilization and minimized network resource consumption. Logesh, K. and Saravanbav, T. P. (2015) proposed that in order to achieve low delay, better bandwidth utilization and low percentage packet loss,RVSP-based resource reservation technique was used in an MPLS-VPN network. TE combined with this RSVP technique was implemented into the MPLS-VPN to improve the performance metrics.

\section{METHOD:}

The work presented in this paper was based on a quantitative research method. This comprised of fieldmeasurement, analytical, experimental and simulation approaches for a typical multimedia Cloud Based MPLS-VPN (CBMV) scenario.

\section{A Characterization of different VPN Networks}

Field measurement and analysis of the MPLS-VPNs were carried out in order to portray their limitations as follows:

Network 1: Internet Solutions Nigeria Limited (ISN)

The first network layout of MPLS-VPN surveyed was the Internet Solutions Nigeria Limited (ISN) whose internal structure is shown in figure 1 below. 


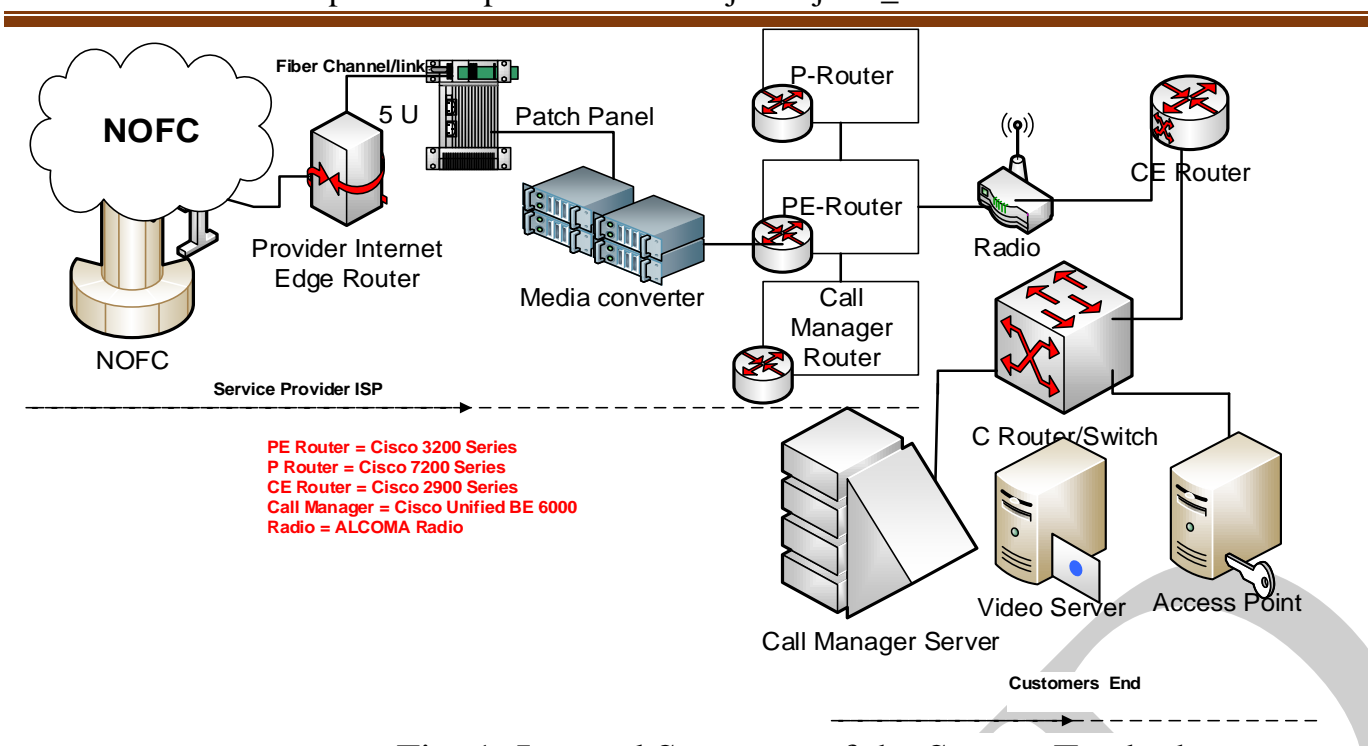

Fig. 1: Internal Structure of the Survey Testbed

This internal structure shows the establishment of a network connection across the Internet via a private network owned by a service provider. From the system, the ISP incorporates Wide Area Network infrastructures through the VPN deployment in a large-scale environment. The system lacks security of the cloud at the core level and a layered framework for building MPLS -VPN that can be used to cater for the needs of various users of the enterprise.

Network 2: Swift enterprise MPLS-VPN

Swift enterprise Data Center Network (DCN) is a leading regional Internet Service Provider (ISP) that offers remote User-to-Site VPN. Table 1 shows the design features and parameters while figure 2 shows the setup of the Swift data center network. This Internet solution uses the global protective agent that allows a remote user to establish a connection between the user and the site using firewall.

Table 1: Design Features/parameters of Swift DCN

\begin{tabular}{|l|l|}
\hline Features/Parameters & Specifications \\
\hline QoS Provisioning & Enabled \\
\hline QoS Profile & Protocol based \\
\hline Link Capacity & $1.5 \mathrm{Mbps}$ \\
\hline Link Connection & Fiber Cables \\
\hline Switch ports & 24 and 48 \\
\hline Terminal nodes LSR & 4 major nodes \\
\hline Network Architecture & Three-Tier \\
& MPLS-VPN Model \\
\hline Packet Rate & 100Mbps \\
\hline Network Server Topology & Servers-In-Rack \\
\hline Remote Server Clustering & Active \\
\hline Storage Area Network (SAN) & Enabled \\
\hline
\end{tabular}

Network 3: UNN Site-to-Site VPN Backbone

The third VPN network considered is the experimental testbed data center network of University of Nigeria Nsukka shown in figure 2. In this case, the network deployed a Site-to- 
Site VPN that connects a central site (UNN) and a remote site (UNN, Enugu campus). This is accomplished using a hub and spoke VPN that connects a central site with multiple remote sites. It has a firewall that uses the IP Security (IPSec) set of protocols to set up a tunnel for the traffic between the two sites. The deployment of the VPN network was arranged in a three-tier pattern. The servers, switches and wireless/wired routers are all connected with Ethernet cables, which are connected to a switch. Each interface is 100Mbps.

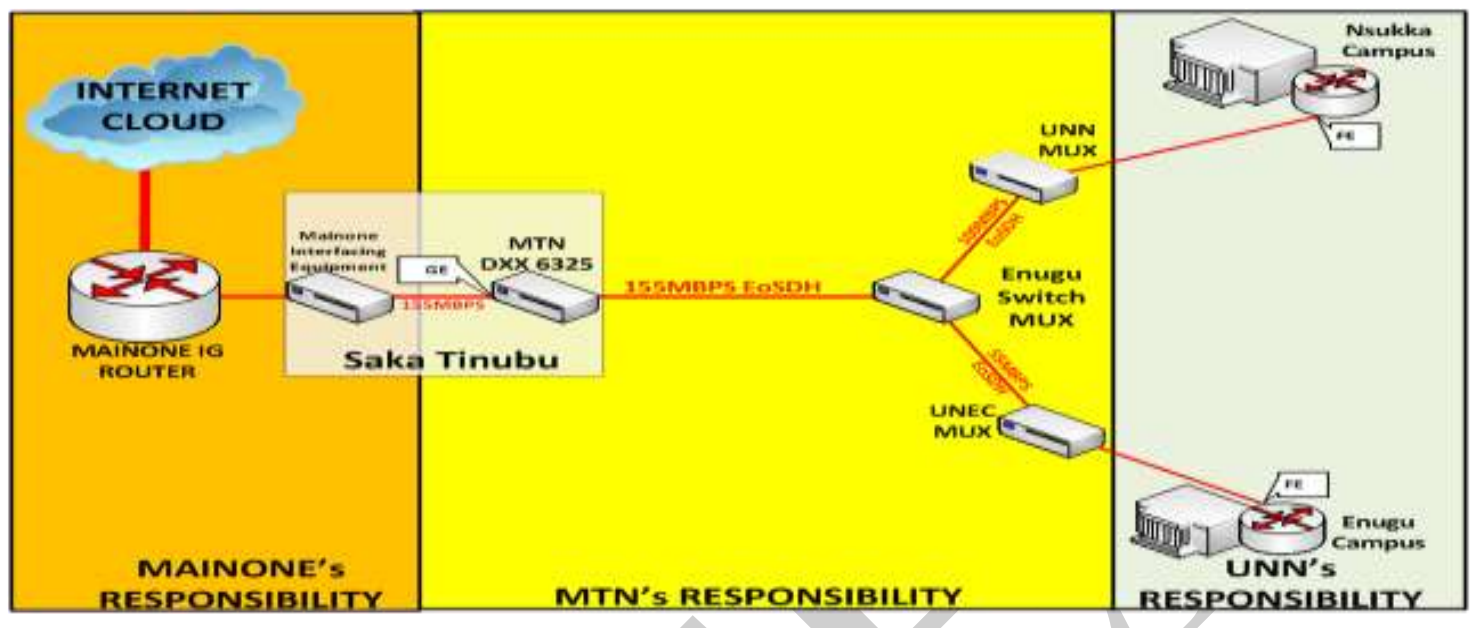

Fig. 2: VPN Structure for UNN Network System

Network 4: NNPC Primary MPLS/VPN Enterprise Wide Area Network

The Nigerian National Petroleum Corporation (otherwise called NNPC or the Corporation) has an MPLS/VPN based Enterprise Wide Area Network. It has its primary and main connectivity between its corporate headquarter (CHQ) and its Strategic Business Units as well as other remote locations. The network provides communication and extends shared services to users enterprise-wide. The required services focus on high-speed Fiber Optics based managed data network services built on Multi-Protocol Label Service (MPLS) and Virtual Private Network (VPN) across the six (6) NNPC Nodal Hubs. The service coverage map of the NNPC locations are as follows: NNPC CHQ Abuja, ITD Centre Lagos, KRPC Kaduna, WRPC Warri, PPMC Area Office Mosimi, ITD Centre Benin and PHRC Port Harcourt. Implementation of end to end Quality of Service (QoS) between the nodal hubs and the head office was done after analyzing the traffic (bulk data, best-effort, transactional data, mission critical, call signaling, voice and video traffic etc.) for traffic prioritization, congestion management and avoidance based on NNPC Preference. Below is the topology of the network as depicted in fig. 3 . 


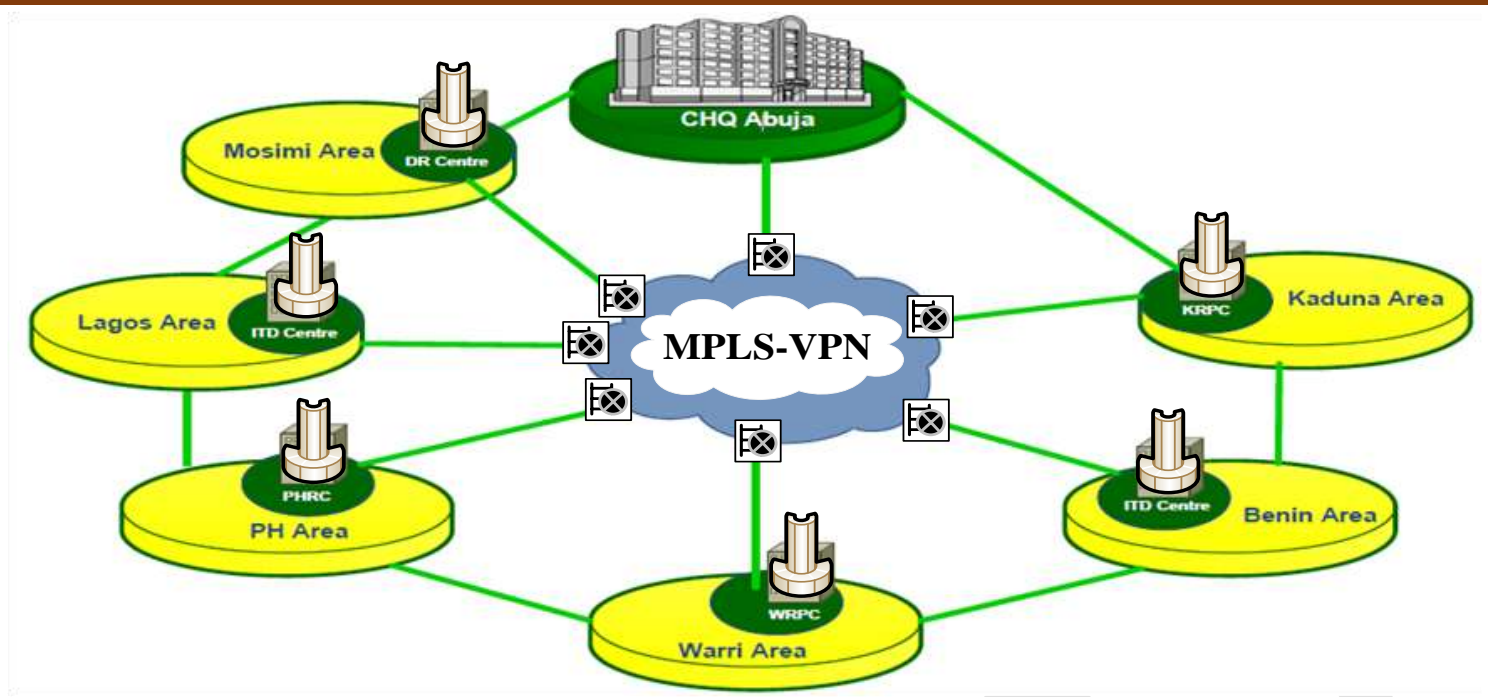

Fig. 3: NNPC MPLS-VPN Structure

Required availability observation was observed. Now, the system offered less than $99.5 \%$ availability KPI. The checks for compliance were done at the discretion of NNPC following the Service Level Agreement template. The network offer management and reporting interface to NNPC on a near $24 \times 7$ basis. This help NNPC keep track of bandwidth usage patterns in each site. The observed bandwidth for each location is as follows: Abuja CHQ 60Mbps, ITD Center/Lagos - 10Mbps, KRPC/Kaduna - 10Mbps, WRPC/Warri - 10Mbps, ITD Center/Benin - 10Mbps, PHRC/ Port Harcourt - 10Mbps, PPMC Mosimi - 20Mbps (NNPC disaster recovery site).

\section{B Deductions from the VPNs Characterization}

From the field work, it was observed that the Layer 3 MPLS-VPNs is the most popular of VPNs deployed for bandwidth utilization. However, ISN was the only network used to analyze the performance of MPLS-VPN. The following are the identified deductions from the testbed structure (ISN) about MPLS-VPN networks.

* The MPLS-VPN (L3 VPNs) cannot offer a good trade-off between deployment cost and transparency to end hosts (It is not cost effective).

* It can support high density services and traffic payload. As a result of massive traffic workload, responsiveness to business need especially in time of speed is very poor for MPLS-VPN due to insecurity of the cloud.

* The ISP have an existing infrastructure with many distributed Points of Presence (PoPs) connected to the backbone, they would prefer to sell virtual connection tunnel rather than physical connections to their customers.

* Usually, VPN network schemes can only be easily deployed once they satisfies the strategies of network device producers and vendors.

* Most enterprise organizations need a robust IP technology that creates a safe and encrypted connection tunnel over the less secure internet. Joint Node Location Problem (JNLP) and Link Connectivity Problems (LCP) were deduced to affect the bandwidth of this network.

According to the topology derived from network 1 (ISN) which is shown in figure 4, when remote users and branch offices want to access corporate applications and other resources these problems constitute the issue of insecurity of the cloud as data travels through the tunnels. 


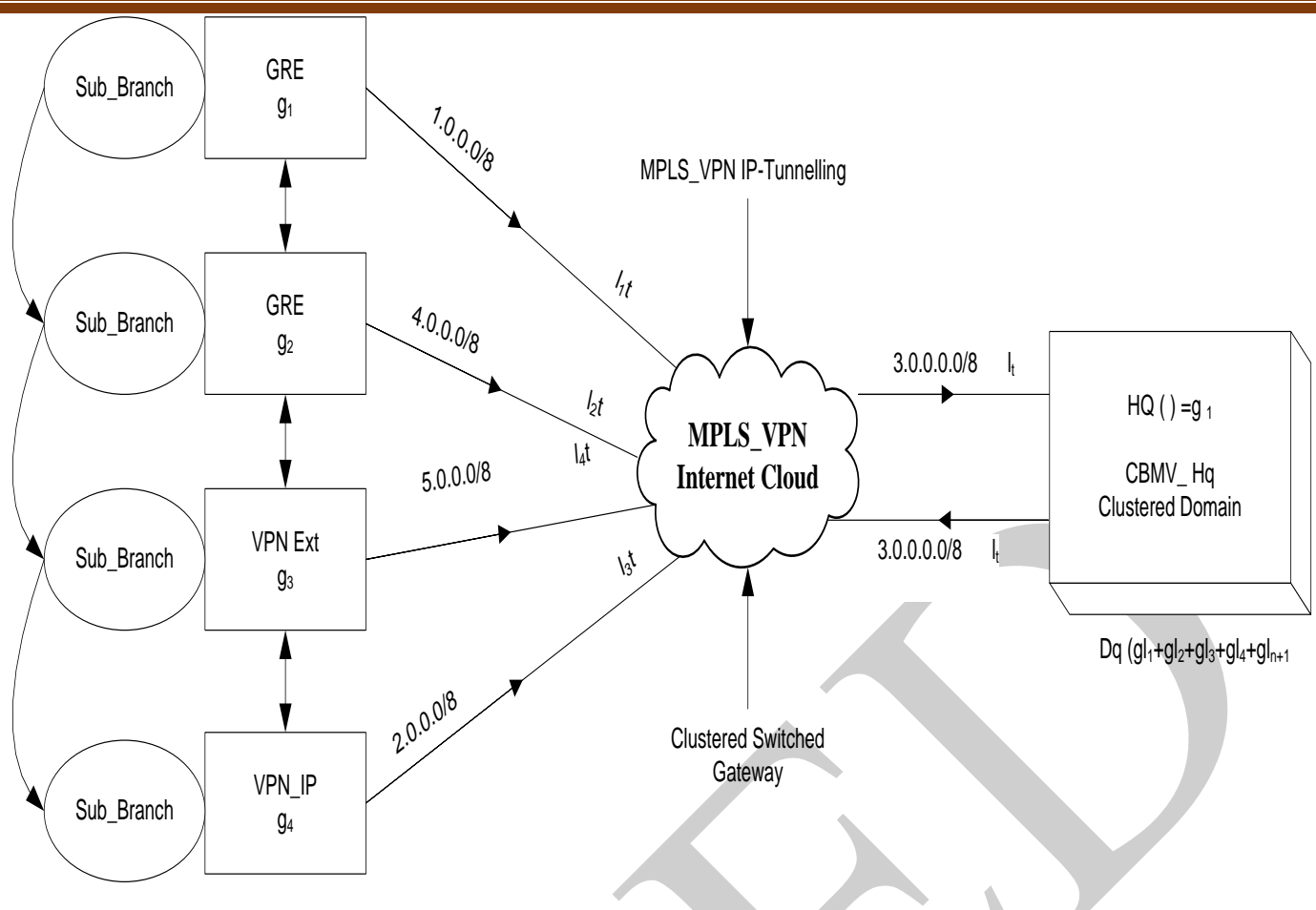

Fig. 4: Topology of MPLS-VPN with JNL and LC problems from ISN

For instance fig.4 above shows typical VPN customers (bank) having several geographically distributed sites and would like to have a unique MPLS-VPN network connecting all of them. It was deduced that there is an insecurity of data at the cloud of the core MPLS. Also there is a link cost associated with the connecting nodes to the HQ core. The interest is in the optimal mapping of IP tunnel which minimizes the access connecting cost (i.e., the connecting cost of the subnet network to access the HQ core network) and the sub branch node placement cost. It also includes the cost of connecting HQ core network nodes.

\section{EXPERIMENTAL SET UP:}

Experimentally a VPN customer Heritage bank headquarter and its' four branches were connected to the ISP network cloud (ISN limited). The experimental call setup flows isshown in the block diagram in fig. 5. The experiment was done during conference meeting held among the bank headquarter and the four branches. Each router in the branch is connected by gigabit Ethernet link with optical fiber between the four different bank branches to headquarter via radio. Data and voice traffic flows were generated during this processes. JPREF used TCP for checking the throughput value of the data traffic of the network, while Video LAN Client (VLC) was used for video streaming and 3CX for making VoIP calls from one end to the other.

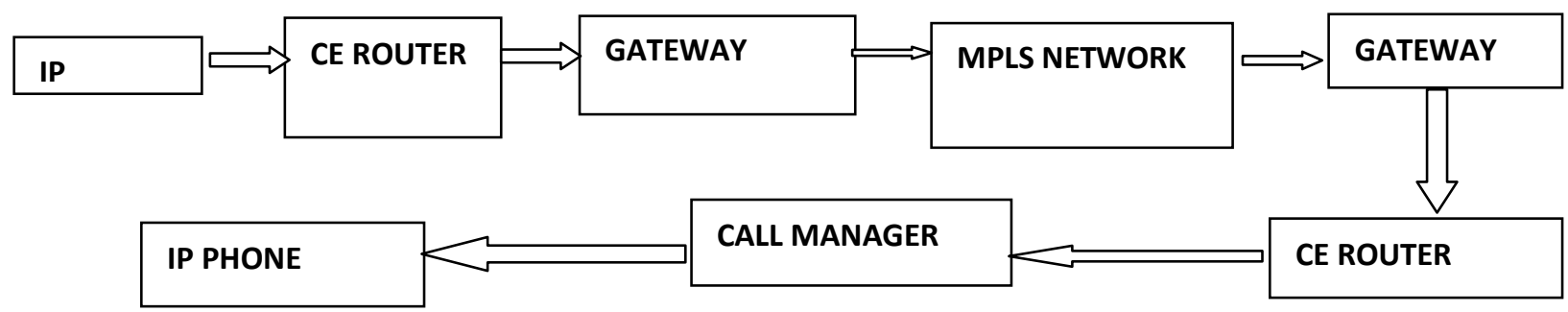

Fig. 5: Block Diagram of Call Setup Flow from one branch to headquarter 
3CX phone system is an IP-based software that uses SIP standard to make voice calls easy to manage. During the course of this experiment VoIP and data traffics were used to analyze the performance.

Packets were transported using specific protocol and all the nodes in the branch locations (Ibadan, Abuja, Kebbi and Owerri) were connected to enable them to access corporate applications in Lagos location (headquarter). Network parameters (packet loss, delay and throughput) for various packet sizes in respect of the locations gotten from experimental view were analyzed in chapter four. Packet loss percentage for various VPN sites were calculated using the formula below

Packet Loss $\%=\frac{\text { Packet sent }- \text { Packet received }}{\text { Packet sent }} * 100$

From these deductions an architectural diagram of an MPLS-VPN was mimicked electronically in a Riverbed tool based on the simulation features shown in the table below

Table 2: Features of Developed MPLS-VPN Network

\begin{tabular}{|l|l|}
\hline Features/Parameters & Specifications \\
\hline Traffic Type & UDP/TCP \\
\hline QoS Profile & Protocol based \\
\hline Link Capacity & $1.5 \mathrm{Mbps}$ \\
\hline Network Architecture & MPLS-VPN \\
\hline Packet Rate & 6 pkt per sec \\
\hline Packet Size & 612 bytes \\
\hline
\end{tabular}

This architectural diagram of a Cloud Based MPLS-VPN in fig. 7 shows a core cloud of an MPLS-VPN that was not secured due to JNLP and LCP.

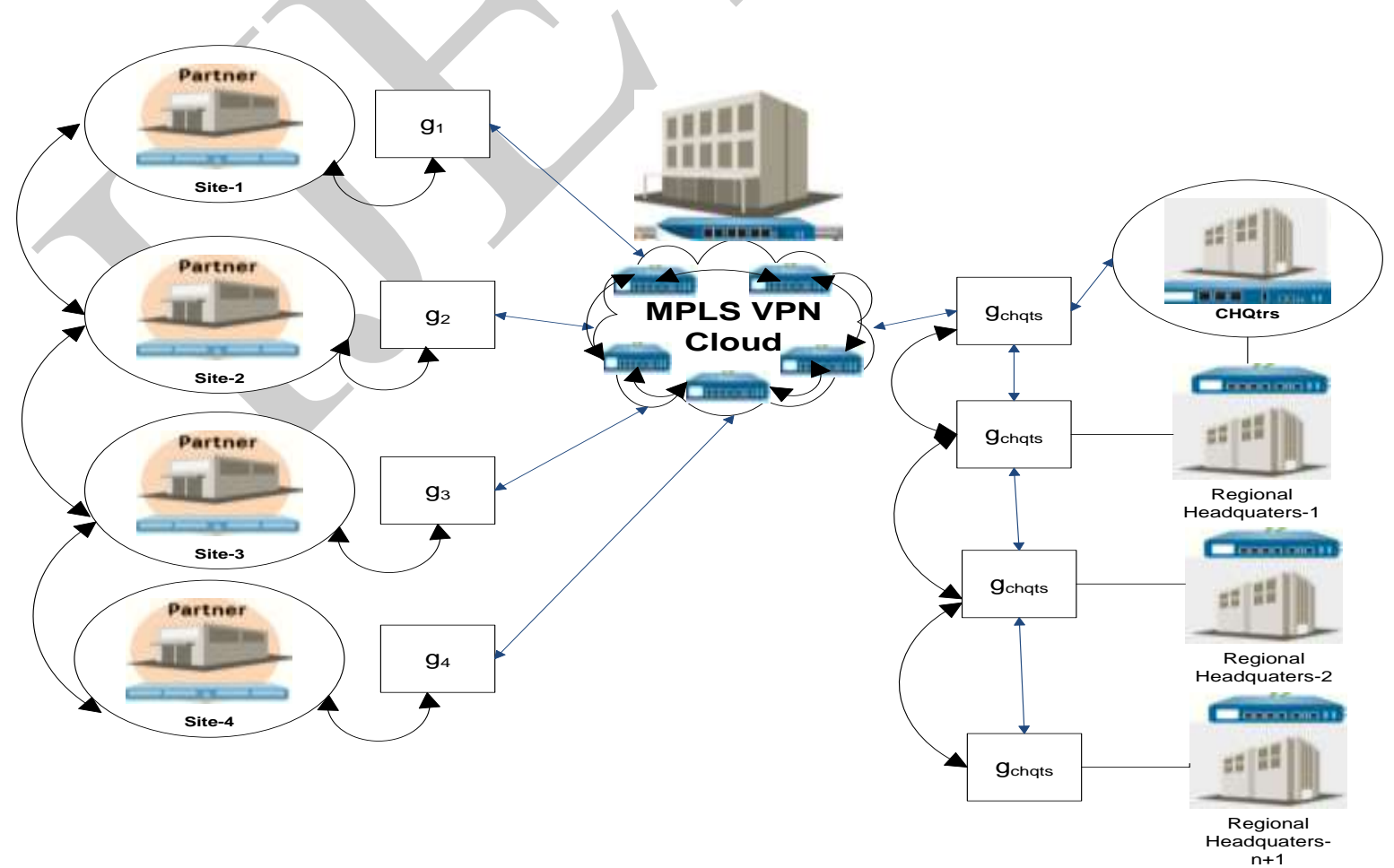

Fig. 6: Developed System Architecture for CBMV Tunneling. 
In the system characterization in fig. 6 , the system provides a robust but simplified scheme to drive a scalable hub and spoke MPLS-VPN (SHS-CBMV) with up to $K=2^{10}$ satellite remote sites/offices.

CBMV label stacking in fig. 7 demonstrates label stacking when multiple MPLS services are enabled in a network shown in fig. 6 .

\begin{tabular}{l|c|}
\hline CBMV Outer Label & $\begin{array}{c}\text { Traffic Engineering, TE } \\
\text { Label }\end{array}$ \\
\hline CBMV Inner Label & $\begin{array}{c}\text { Interior Gateway Routing } \\
\text { Protocol, IGP [OSPF] Label }\end{array}$ \\
\hline CBMV Lable \\
\hline SDA label \\
IP Header
\end{tabular}

Fig. 7: CBMV label stacking for optimal traffic Tunneling.

The CBMV service in Figure 8 allows for the support of the user multicast traffic in a BGPVPN environment. IP-header gives support to multicast video, voice, and data traffic within the VPN which was used to determine the impact of QoS metrics on MPLS-VPN performance.

\section{RESULTS ANALYSIS}

According to ITU standard, the allowable threshold durations for the network performance metrics are depicted in table 3. The Internet Solution of Nigeria Limited (ISN) structure testbed studied was used to obtain specific network parameters.

Table 3: ITU QoS Standard for Network Quality Performance (Chor, et.al2015)

\begin{tabular}{|l|l|l|l|}
\hline Network Parameter & Good & Acceptable & Poor \\
\hline End-to-End delay (msec) & $0-150$ & $150-300$ & $>300$ \\
\hline Delay variation (msec) & $0-20$ & $20-30$ & $>30$ \\
\hline Packet Loss (\%) & $0-0.5$ & $0.5-1.5$ & $>1.5$ \\
\hline Throughput (Mbps) & $0-50$ & $50-144$ & $>144$ \\
\hline
\end{tabular}

The results of Heritage bank branches of four different locations with 2028Kbytes bandwidth between PE and CE routers were analyzed. This was done considering file transfer protocol for voice and data traffic that run on the ISN MPLS backbone. Internet Control Message Protocol was used to ascertain the throughput, packet loss and delay metric values against the packet rates of the transmission. Its echo time when there is no response was set to maximum of 2 seconds for the four different locations.

\section{Throughput Response against Packet Rate}

The graphical representation that presented the throughput response obtained from the four different VPN customers as against packet rate when they were communicating remotely to headquarter via VoIP. 
Theoretically in a communication network, throughput as a metric varies proportional to the packet rate. However, from the experiment, it was observed that the throughput value from Ibadan and Abuja VPN customers were constant between the intervals of $100 \mathrm{Kbps}$ to $700 \mathrm{Kbps}$ packet rate. This was because the network radio link between the two different locations and Lagos (headquarter) has a smaller distance to compare with Kebbi and Owerri sites that uses two different transmission media (radio and fiber) along their distances. From the fig. 8, the study concluded that the experimental values of throughput does not vary proportionally to the packet rate. Hence the QoS provisioned was affected and it needs to be improved.

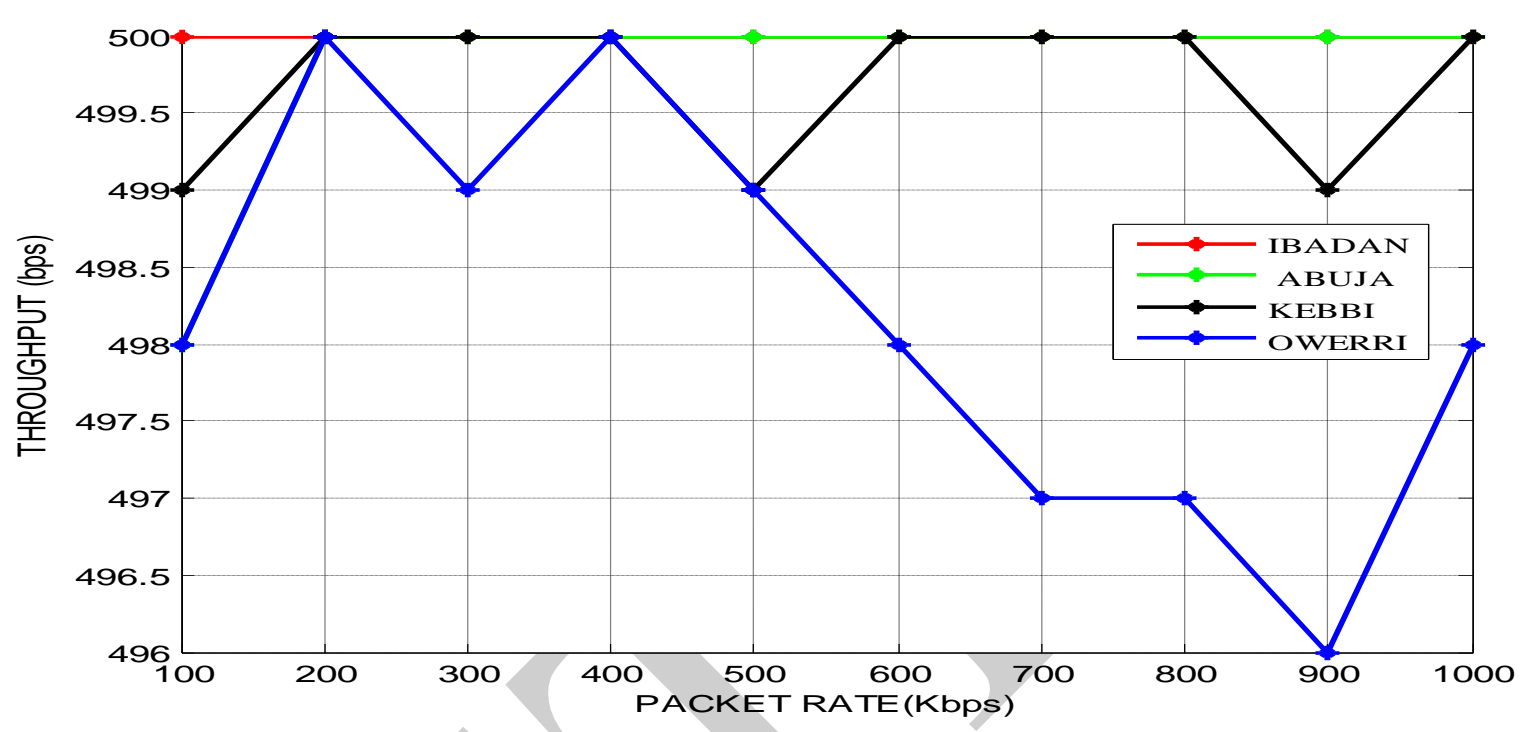

Fig.8: Graph of Throughput Response against Packet Rate for the four VPN users

\section{Packet Loss Response against Packet Rate}

In a practical WAN network like MPLS-VPN, the expected packet loss probability is $0.1 \%$ and this means that there should only be one loss of packet in every 1000 packets sent. Results of figure 9 shows that the average percentage loss of packet for the four different VPN were approximately $2 \%$.

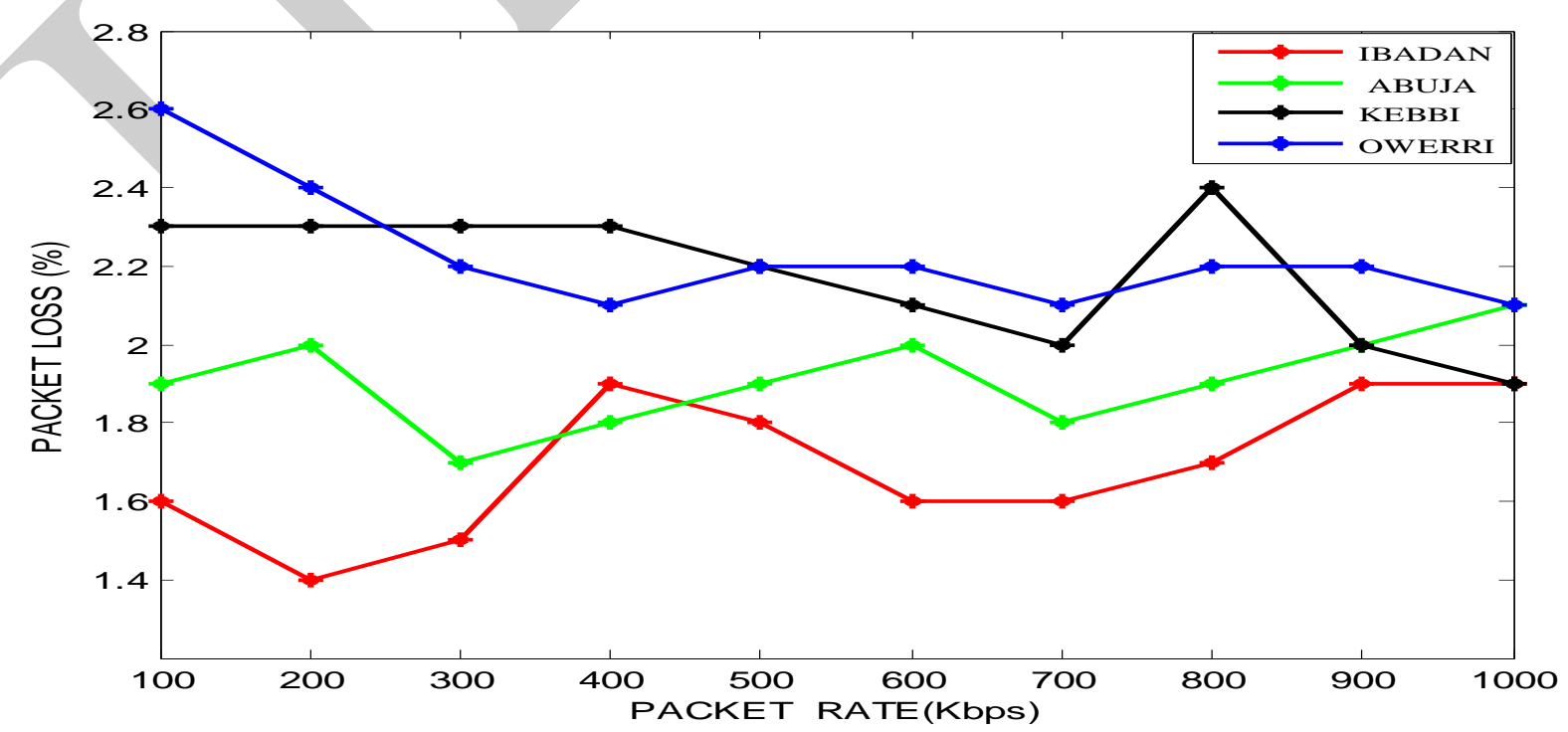

Fig. 9: Graph of Packet Loss Percentage against Packet Rate from the four different VPN Users 
The percentage rate of packet loss varies randomly with the packet rate for the four different VPN customers. From fig. 9, it was observed statistically that approximately 20 packets were lost out of the transmitted data from the four different VPN branches. At 400Kbps and $800 \mathrm{Kbps}$ packet rate, the percentage packet loss increases during the data transmission from Ibadan and Kebbi VPN locations respectively unlike other customers. This was because of the sudden changes in the network routing protocol as a results of error that occurred within the period of transmission. These packet loss variation causes video pixilation and hanging of voice calls at the destination VPN customer (Lagos).

\section{End-to-End DelayResponse against Packet Rate}

Similarly in data communication, the amount of traffic flow (number of bit) in a transmission channel between source and destination is expressed theoretically as

$\mathrm{N}=(D) *[R](1.2)$

Where $(D)$ is the delay time in second taken for the data to travel from source to destination and $[R]$ is the flow rate of packet in bits per second

End-to-End delay response inversely related to the packet rate, but at $400 \mathrm{Kbps}$ rate there was a change in the variations especially during traffic transmission from Ibadan and Abuja to destination (Lagos). A sharp increase of the delay metrics after 400Kbps was induced by router buffer. This buffering act occurred remotely in the Ingress router at the two locations. Recall theoretically that end-to-end delay comprises of processing, queueing, transmission and propagation delays. It was observed initially during the traffic transmission from Ibadan that there were negligible values of processing and transmission delays that is why delay varies correctly with packet rate.

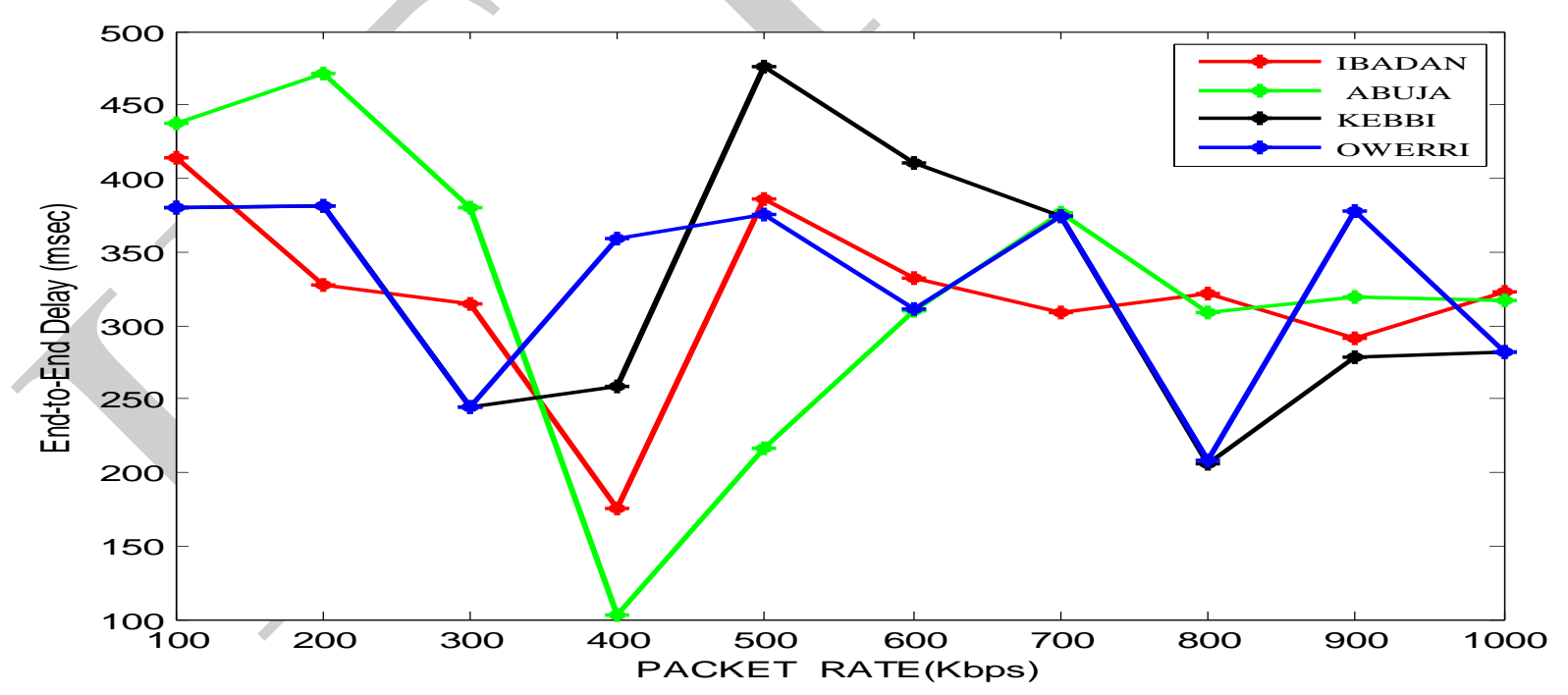

Fig. 10: Graph of End-to-End Delay Response against Packet Rate from the four different VPN Users

For all the packet rates with 1000 bytes packet sent from the four different sources (various locations) to destination (headquarter), the average delay, packet loss and throughput as shown in figs. 8 to 10 were observed to be greater than $300 \mathrm{msec}, 1.5 \%$ and $144 \mathrm{bytes} / \mathrm{sec}$ respectively which are the ITU threshold values shown in table 3 . These metrics were within the poor threshold value as compared with the ITU values. All the metric responses were 
insufficient for cloud based MPLS services provisioning thereby causing distortion at critical times for time-sensitive and best-effort traffic on the network.

\section{CONCLUSION:}

Deterioration occurs in data network when its metrics fall below the allowable range. There was existence of Joint Node Location Problem (JNLP) and Link Connectivity Problem (LCP) as a result of insecurity challenges in the cloud of MPLS-VPN.These poor metrics lead to insecurity of data, causing dragging / hanging of window operating system during FTP applications, too much delay and drop of calls in VoIP.In summary, the characteristics of the network infrastructure has revealed that MPLS-VPN lacks an improved QoS provisioning. Future work will include the development of a load balancingvirtualizationmodelthat will help in the improvement of the performance of MPLS-VPN.

\section{REFERNCES:}

1. Abdul-Bary, R. S. and Omar-Kh, S. A. (2014). "Performance Analysis of Multimedia Traffic over MPLS Communication Network with Traffic Engineering". International Journal of Computer Networks and Communication Security. Vol. 2, No. 3, pp. 93 -101.

2. Aggarwal, J. and Dhall, A. (2015). "Performance Analysis of MPLS over VoIP". International Journal of Science Engineering and Technology Research. Vol.4, No.6, pp. $1896-1901$.

3. Ahmad, S., Hamdani, W. A., Magray, M. H. (2015). "Performance Evaluation of IPv4 and IPv6 over MPLS using OPNET. International Journal of Computer Applications. Vol. 25, No. 3, pp. $34-38$.

4. Almofary, N. H., Moustafa, H. S. and Zaki, F. W. (2013). "Scalability Aspects in BGP/ MPLS - VPN". International Journal of Modern Engineering Science. Vol. 2, No. 1, pp. $17-27$.

5. Chor, A.L, Dai, W. E. and Zhang, C.C. (2015). Performance Analysis of VoIP over Network. http:// www.freescale.com/files/wireless-comm/doc/white-paper

6. Cole. J, E., Thain, W. E. and Fallon, T. (2015). "Modeling Multi-Protocol Label switching Networks in the Laboratory". $122^{\text {nd }}$ ASEE Annual Conference and Exposition.

7. Efendi, R. (2012). "A Simulation Analysis of latency and Packet Loss on Virtual Private Network through Multi Virtual Routing and Forwarding". International Journal of Computer Applications. Vol. 60, No. 19, pp. $50-56$.

8. Kale, N. N. and Waichol, S. A. (2014). "Performance Analysis of MPLS Network with Traditional IP Network in Service Provider Environment”. International Journal of Advanced Research in Computer Engineering \& Technology. Vol. 3, No. 4, pp. 1311 1316.

9. Kundu, R., Kumar, A. and Wason, A. (2014). "Performance Analysis and Improvement on QoS of MPLS networks". ICRTEDC Vol.1, No.2, pp. 227-228.

10. Logesh, K. and Saravanabava, T. P. (2015). "QoS Provisioning for Multimedia Traffic on MPLS-VPN with Traffic Engineering”. International Journal of Electrical Electronics and Data Communications. Vol. 3, No. 4, pp. 51 -57. 
11. Lutfullah, K.A.M., Hassan, M. Z., Amin, M. R., Rahman, M. A., and Kabir, A. H. (2008). "Performance Analysis and the Study of the behavior of MPLS Protocols". International Conference on Computer and Communication Engineering, pp.13-15.

12. Mamum, A. A., Sheltami, T. R., Ali, H. and Anwar, S. (2016). "Performance Evaluation of Routing Protocols for Video Conference over MPLS-VPN network". Journal of Ubiquitous Systems \& Pervasive Networks. Vol. 7, No. 1, pp. $1-6$.

13. Mishra, R., and Ahmad, H. (2014). "Comparative Analysis of Conventional IP Network and MPLS Network over VoIP Application". International Journal of Computer Science and Information Technologies. Vol. 5, No. 3, pp. $4496-4499$.

14. Sumithra, A. and Devaraj, B. (2014). "Creation of Virtual Private Network over MPLS Network for Voice over IP”. International Journal of Research. Vol.1, No. 9, 1206-1212.

15. Yunos, R., Ahmad, A., Noor, N. M., Saidi, R. M., and Zainol, Z. (2013). "Analysis of Routing Protocol of VoIP VPN over MPLS Networks. IEEE Conference on Systems, Process, and Control. pp. $139-143$. 